\title{
A novel designed in-ceram zirconia fixed dental prosthesis: case report
}

\begin{abstract}
Objectives: This clinical report describes a relatively less-invasive, functional and more conservative hybrid fixed prosthodontics treatment option for a patient with missing tooth combining laminate veneering abutment while the other abutment was fully covered.
\end{abstract}

Patient and methods: A female patient with a missing maxillary left central incisor was less-invasively treated with a FDP with an all ceramic In-Ceram zirconia restoration covering the right lateral and laminating the left central.

Results: The restoration has served the patient aesthetically and functionally for 5 years, seemingly without discomfort, and it has not required any maintenance. The patient has kept up with her oral hygiene.

Conclusions: Although additional clinical experience is necessary, this new design can be used in a special situation.
Volume 8 Issue 5 - 2017

\author{
Mohamed Hamed Ghazy \\ Department of Fixed Prosthodontics, Mansoura University, \\ Egypt
}

Correspondence: Mohamed Hamed Ghazy, Department of Fixed Prosthodontics, Mansoura University, Egypt,

Email mghazy@mans.edu.eg

Received: October II, 2017 | Published: November 01, 2017

\section{Introduction}

Nowadays a trend to shift toward metal-free restorations has been observed in the dental field because from a purely cosmetic standpoint, the value of the appearance of one's teeth has taken on a greater importance in today's society therefore, all ceramic restoration has became the mainstay of aesthetic dentistry in the last few years offering superior aesthetics combined with high bio-compatibility, ever increasing strength and long-lasting restorations. Therefore several types of all ceramic systems have been developed. ${ }^{1-4}$ Among them, In-Ceram Zirconia (VITA Zahnfabrik) which developed as a modification of the original In-Ceram Alumina system, with an addition of $35 \%$ partially stabilized zirconia oxide to the slip composition to strengthen the ceramic. ${ }^{5}$ The available fixed dental prosthesis (FDP) treatment options for missing anterior tooth include implant supported crown, traditional and resin-bonded teeth supported FDP. Every treatment modality offers some advantages and disadvantages but still the golden role for the dentist and patient is that, whenever possible conservation of tooth structure is a goal during teeth preparation.

\section{Case report}

A 22-years-old female patient presented to the dental clinic with a chief complaint of missing maxillary right central incisor tooth and misaligned (rotated and palatally inclined) maxillary left central anterior (Figure 1). Past dental history revealed that the maxillary right central incisors had been previously traumatically fractured and restored several times before extraction and right lateral incisor have a successful root canal treatment. The patient's main treatment goals were to align her maxillary teeth in addition to replace the missing tooth. The treatment planning options was discussed with the patient as replacing the missed central with implant supported crown and constructing all ceramic restoration on the right lateral and left central incisor to adjust its alignment, orthodontic alignment for the left central followed by constructing a fixed fixed conventional or resin bonded dental prosthesis using the right lateral and left central as abutments, or constructing a hybrid fixed dental prosthesis by full covering the right lateral and laminating the left central incisor. The suggested pretreatment orthodontic option was declined by the patient after an orthodontic consultation and she made her decision to change the appearance of her maxillary teeth through the tooth supported fixed restorations only to save time and cost of the treatment. Alginate primary impression was taken for the patient and diagnostic cast was poured. The diagnosis of the case was confirmed by a preoperative radiograph and study model to calculate the labial thickness needed to realign the central incisors in the arch contour. The teeth color was selected be 2M1 using vita 3D master shade guide (Vita). Because of the palatal inclination maxillary left central incisor, the decision was made to construct all-ceramic fixed dental prosthesis replacing the missed central by preparing the left central from the labial surface only like that of the laminate veneer preparation and prepare the endodontically treated right lateral incisor to be fully coverage. Two $\mathrm{mm}$ reduction was done for the incisal edge of the right lateral. Mesial and distal surfaces were reduced to a. ${ }^{2-5}$ degree taper and shoulder of $1 \mathrm{~mm}$ at the margin. Palatal surface was reduced in two planes. First a cingulum shoulder is placed with a flat ended tapered diamond bur then a wheel shaped diamond bur is used to reduce the palatal concavity. Facial reduction is performed with a coarse, flat ended diamond bur using smooth, controlled, sweeping motion. The incisal two-thirds of the facial surface inclined palatally to provide uniform ceramic thickness and ensure suitable esthetics. ${ }^{6}$ For the right central, the labial surface was reduced $0.5 \mathrm{~mm}$ only by the use of 3 wheel depth cutter first then completed using tapered stone. The preparation ended at the incisal edge, just before the distal contact area and beyond the mesial one. The completed preparation was smoothened with finishing stones and any sharp angles were removed (Figure 2). Retraction cord (ultradent, Inc. 050W. 10200S. South Jordan, UT84095) was adopted 
along the cervical finish lines of the prepared teeth then impression of heavy and light bodies were taken with polyvinylsiloxane (Virtual, Ivocalr Vivadent, Schaan, Liechtenstein) and poured into extra hard stone (Vel-Mix Stone; Kerr. Basel, Switzerland). Provisional cantilever acrylic fixed restoration was fabricated and cemented to the lateral incisor with temporary cement until the time of the try in (Figure 3). The In-Ceram zirconia substructure was fabricated on the working cast following the manufacturer's recommendations and checked in patient mouth for verification of its seating, adaptation, contour retention and stability (Figure 4). Any fine adjustment needed was done until the restoration became satisfactory then it was sent to the laboratory for facial surface veneering with the veneering ceramic (VM9, Vita, Zahnfabrik-Bad Säckingen, Germany). The final restoration was tried in again to check its final contouring and alignment in the maxillary arch in addition to color matching with their adjacent teeth color before cementation (Figures 5-7). The intaglio surfaces of retainers of the completed restorations were airborne particle-abraded with $50 \mu \mathrm{m} \mathrm{Al} 203$ then, cleaned and decontaminated with K-Etchant Gel (Kuraray) to prepare for cementation. Clearfil Ceramic primer (Kuraray) was placed on the intaglio surface of the restoration. The two abutment teeth were etched with $37 \%$ phosphoric acid for 15 seconds then rinsed with sterile water. ED Primer A and B was mixed one drop each in the well supplied with the kit and applied to the abutment teeth using a disposable brush tip, left in place for 60 seconds, then dried with a gentle air. The hybrid FDP was bonded to the etched prepared teeth with the use of a dual polymerizing composite (PANAVIA F 2.0 TC, Kuraray, and Japan). During the setting of the luting resin, the FDP was retained by the operator hand for 3 minutes and the excess composite was removed with sponge pellets. The margins were light-cured for 20seconds in several areas and air-blocking gel (Oxyguard II, Kuraray) was then applied during the setting time of the resin cement (7minutes). After polymerization was completed, excess cement was removed from the margins with a periodontal scalers and the occlusion was confirmed in centric, protrusive, and lateral excursions. The restorations were evaluated clinically and radiographically with regard to changes soon after cementation and through 6 month periodic recalls using California dental association criteria. After a five years follow-up, the restorations and cement/teeth interface were clinically perfect with no chipping, fractures or discoloration.

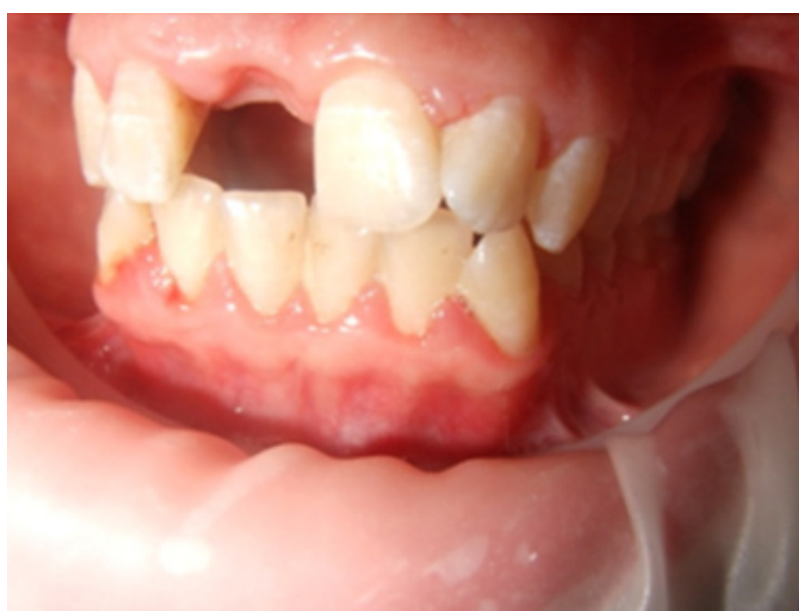

Figure I Before preparation.

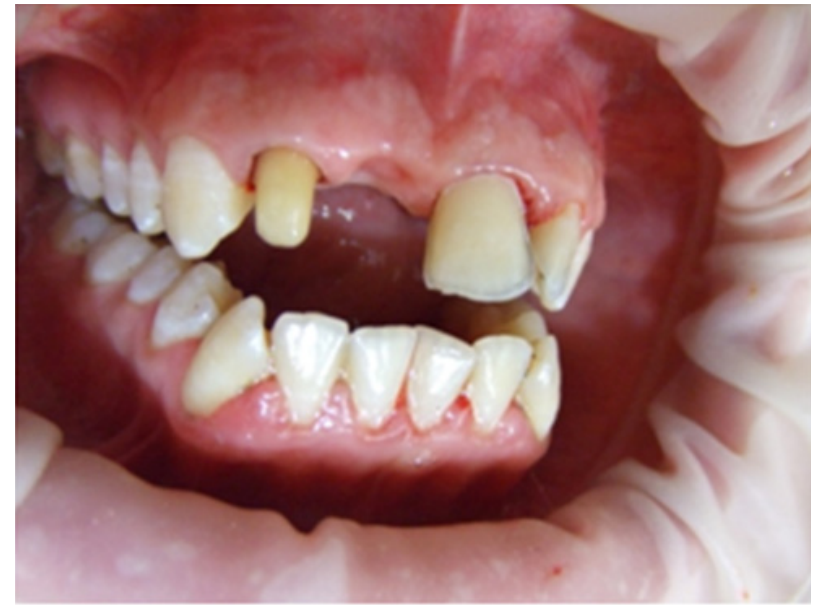

Figure 2 Final preparation after gingival retraction.

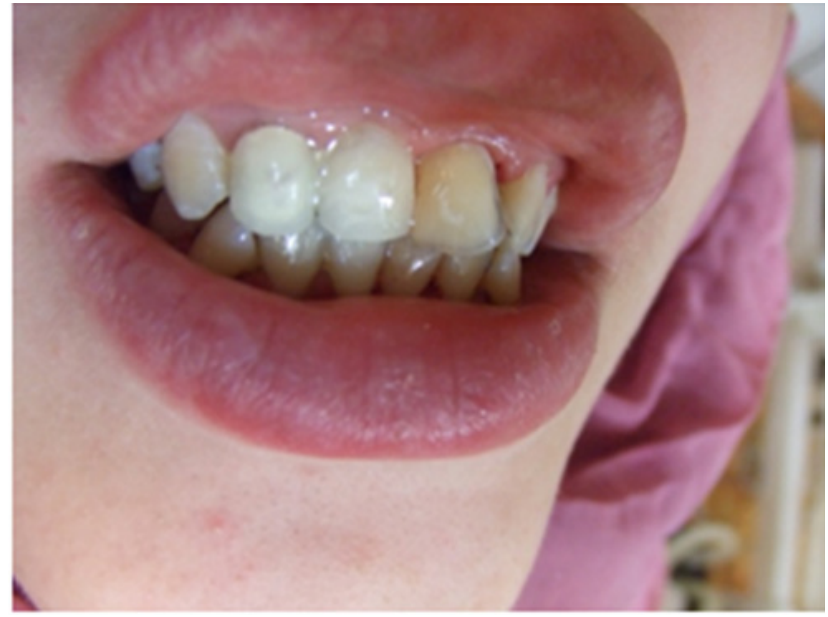

Figure 3 Provisional FDP in place.

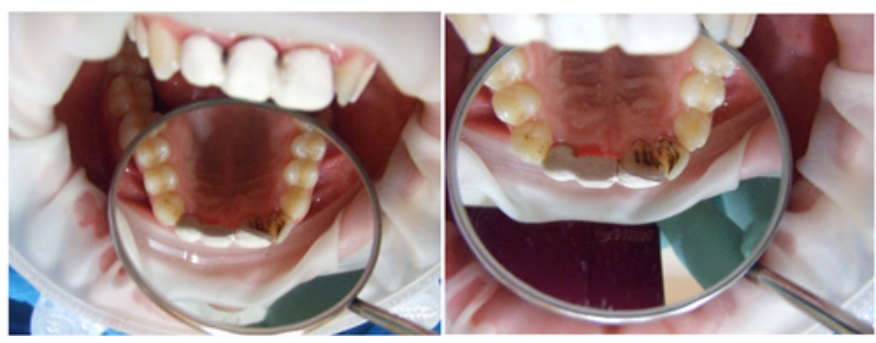

Figure $4 \mathrm{ln}$-Ceram zirconia core try in labial and palatal views.

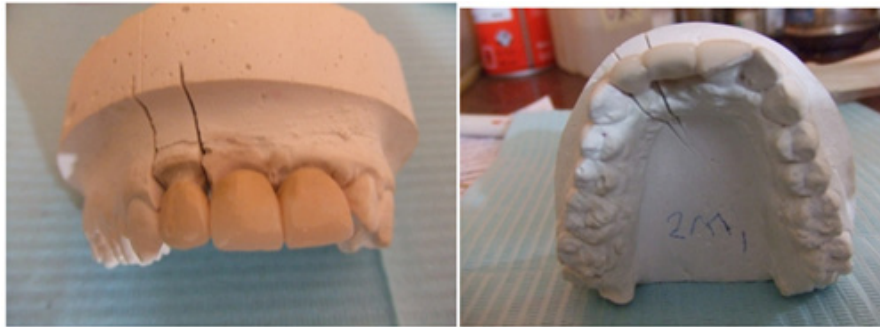

Figure $\mathbf{5}$ Labial and incisal views of the final restoration on the working cast. 

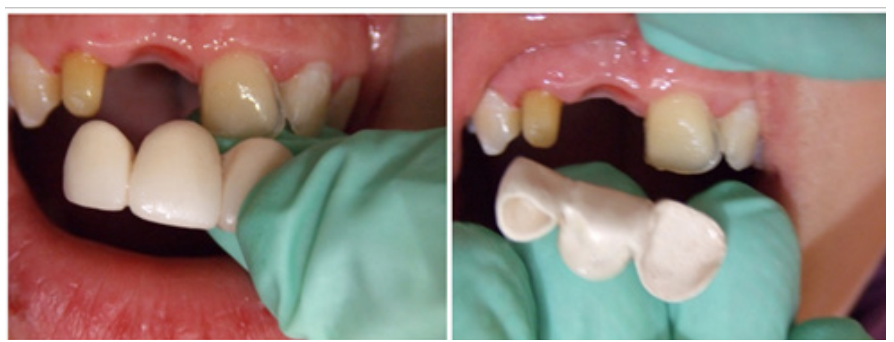

Figure 6 Labial and palatal views of the final restorationn, try in stage.

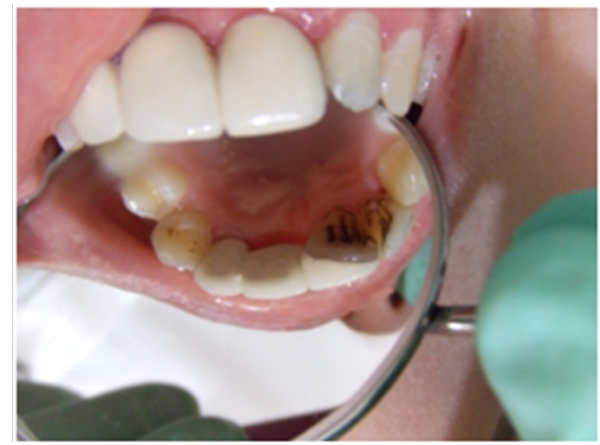

Figure 7 Palatal view of the final restoration, try in stage.

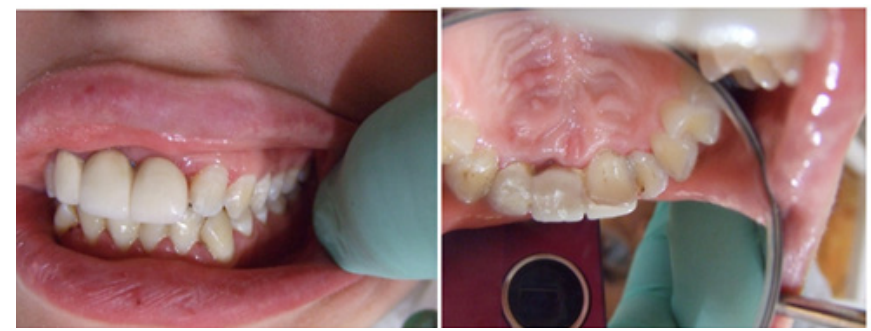

Figure 8 Follow up 6 months.

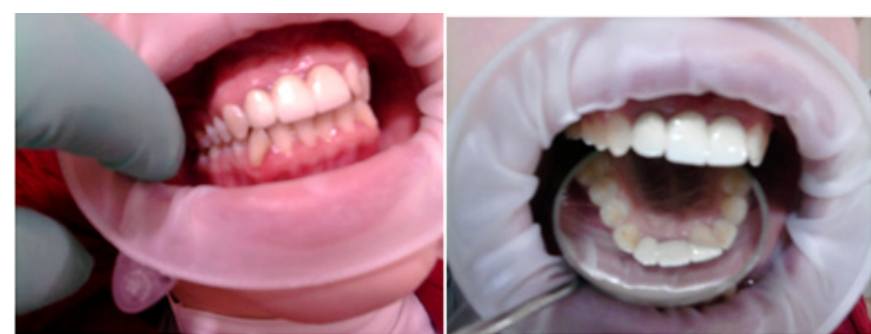

Figure 9 Follow up one year.

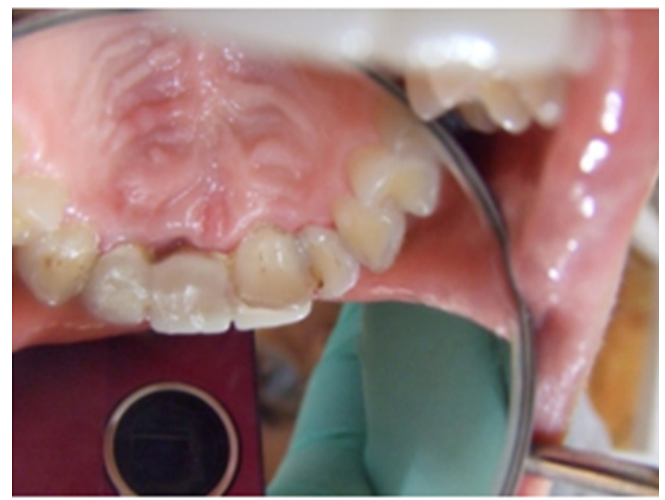

Figure 10 Palatal view, 5 years follow up.

\section{Discussion}

The first choice for replacing missing anterior tooth would be implant supported crown restoration. Unfortunately this option is not always feasible because of medical reasons, habits, treatment cost, patient desires and inadequate bone not amenable to grafting. The most conservative technique of restoring the missing central incisor for this patient would have been implant supported crown and a laminate veneering the right central to correct its alignment in addition to full covering the endodontically treated right lateral tooth. However, the patient refused implant placement, which would have required more time and cost than the conventional replacement with a fixed restoration. The laboratory technician thought correction the palatal inclination of the right central could be achieved with increasing the labial thickness of the restoration with high strength ceramic restoration therefore, the final decision was to construct a FDP from a high-strength ceramic on with a new design full covering the maxillary left lateral incisor with the right central as a pontic and place a bonded labial wing on the upper left lateral incisor. In-Ceram Zirconia (Vita Zahnfabrik, Bad Sackingen, and Germany) was selected as the high strength ceramic material of choice for that case because of its recorded high strength and acceptable final esthetic result. ${ }^{7}$ In this case the right central incisor was cantilevered on the FDP without fear of fracture at the connector area which is considered one of the main causes cantilever failure, this is due to the ability of increasing the thickness of the connector area above the recommended minimum dimension of an anterior 3-unit all-ceramic FDP of Y-TZP being $3 \mathrm{~mm}$ in incisal cervical direction and $2 \mathrm{~mm}$ in labio-lingual direction, which is gained simultaneously during increasing the thickness of facial wing of FDP to correct the palatal inclination of the right central incisor. ${ }^{8}$ Various techniques have been introduced to provide a mechanical attachment between resin and ceramic. ${ }^{9}$ Hydrofluoric acid and subsequent placement of a silane coupling agent will increase the bonding strength to feldspathic ceramic. However, this technique is not applicable to the high-alumina or zirconia ceramic materials because these do not contain silicon dioxide. High-strength ceramics (alumina and zirconia) are more chemically stable than silica glasses and ceramics that contain silica. This stability and glass-free aspect does not allow the zirconia to be treated with hydrofluoric acid etching and silanization to create a stable bond..$^{10}$ One of the most frequently used resin cements in dentistry is Panavia F 2.0. It consists of a bifunctional monomer, 10-methacryloyloxydecyldihydrogenphosphate (MDP). ${ }^{11}$ Matinlinna et al. ${ }^{12}$ found that the combination of airborne-particle abrasion and (MDP) monomer is the recommended method for bonding resin composites to zirconia.

\section{Acknowledgments}

None.

\section{Conflicts of interest}

The authors declare that there is no conflict of interest.

\section{Funding}

None.

\section{References}

1. Denry I, Holloway JA. Ceramics for dental applications: a review. Materials. 2010;3(1):351-368. 
2. Conrad HJ, Seong WJ, Pesun IJ. Current ceramic materials and systems with clinical recommendations: a systematic review. $J$ Prosthet Dent. 2007;98(5):389-404.

3. Della Bona A, Kelly JR. The clinical success of all-ceramic restorations. J Am Dent Assoc. 2008;139(Suppl):8S-13S

4. Al-Amleh B, Lyons K, Swain M. Clinical trials in zirconia: a systematic review. J Oral Rehabil. 2010;37(8):641-652.

5. Sundh A, Sjogren G. A comparison of fracture strength of yttriumoxidepartially-stabilized zirconia ceramic crowns with varying core thickness shapes and veneer ceramics. $J$ Oral Rehabil. 2004;31(7):682-688.

6. Munson CM. Anterior crowns : principles and practice. 3.Prepration of vital teeth. Dent Update. 1985;12(1):25-28.

7. Bello A, Jarvis RH. A review of esthetic alternatives for the restoration of anterior teeth. J Prosthet Dent. 1977;78(5):437-440.
8. Bahat Z, Mamood DJ, Vult von Steyern P. Fracture strength of three-unit fixed partial denture cores (Y-TZP) with different connector dimension and design. Swed Dent J. 2009;33(3):149-159.

9. Ozcan M, Vallittu PK. Effect of surface conditioning methods on the bond strength of luting cement to ceramics. Dent Mater. 2003;19(8):725-731.

10. Aboushelib MN, Matinlinna JP, Salameh Z, et al. Innovations in bonding to zirconia-based materials: part I. Dent Mater. 2008;24(9):1268-1272.

11. Ozcan. Effect of various surface conditioning methods on the adhesion of dual-cure resin cement with MDP functional monomer to zirconia after thermal aging. Dent Mater J. 1988;27(1):99-104.

12. Matinlinna JP, Heikkinen T, Ozcan M, et al. Evaluation of resin adhesion to zirconia ceramic using some organosilanes. Dent Mater. 2006;22(9):824-883. 